\title{
Immunology and Cell Biology of Parasitic Diseases
}

\author{
Luis I. Terrazas, ${ }^{1}$ Abhay R. Satoskar, ${ }^{2}$ and Jorge Morales-Montor ${ }^{3}$ \\ ${ }^{1}$ Laboratorio de Inmunoparasitología, Unidad de Biomedicina, Facultad de Estudios Superiores-Iztacala, \\ Universidad Nacional Autónoma de México, 54090 Tlalnepantla, MEX, Mexico \\ ${ }^{2}$ Department of Microbiology, Center for Microbial Interface Biology, The Ohio State University, 484 West 12th Avenue, \\ Columbus, OH 43210, USA \\ ${ }^{3}$ Departamento de Inmunología, Instituto de Investigaciones Biomédicas, Universidad Nacional Autónoma de México, AP 70228, \\ 04510 México, MEX, Mexico
}

Correspondence should be addressed to Luis I. Terrazas, literrazas@campus.iztacala.unam.mx

Received 31 December 2010; Accepted 31 December 2010

Copyright (C) 2010 Luis I. Terrazas et al. This is an open access article distributed under the Creative Commons Attribution License, which permits unrestricted use, distribution, and reproduction in any medium, provided the original work is properly cited.

This special issue of Journal of Biomedicine and Biotechnology focuses on research in immunology and cell biology of parasitic diseases carried out worldwide. In this issue, you will find contributions by world leaders and also have the opportunity to read some of the hottest topics in immunoparasitology and cell biology of parasites, with emphasis on parasites that are responsible for significant morbidity and mortality in the world. The question is: why this special issue was devoted to present these works? There are three main reasons for this; the first is related to the fact that this research field has made an impressive progress during the past decade, supported by numbers showing that the proportion of the world's scientific publications increased almost $100 \%$ in the past two decades. The number of Ph.D. scientists awarded in the world, and particularly in underdeveloped countries, has also risen significantly in recent years, particularly in the field of parasitology. Notably, albeit at different rates, a growing number of scientists are dedicated to this field in developing as well as developed countries. Indeed, in underdeveloped countries, general efforts have been made to promote the development of this science during the last decade, mainly due to the fact that parasites are indeed a significant health problem. The second reason is that a quarter of world population is at risk or exposed to different parasites, that endanger and compromise health of these individuals. Particularly, underdeveloped countries are places where ecology, climate, economic, and social status allow establishment of a wide variety of parasitic diseases. Moreover, globalization with increased migration between developed and underdeveloped countries favor the spread of many parasitic diseases worldwide. In addition to the migration, our unquestionable global climate change is also contributing to emergence of parasitic diseases in previously nonendemic areas. Thus, a great number of scientists and institutions in the world are really interested and involved in parasitology research. The third reason is that we have passed the first decade of the 21th century without development of a confident vaccine against any parasitic disease that affects humans, even though great efforts have been made for many institutions. This fact propels us to do more in-depth research in order to know closer our enemies and, in some moment, reach the adequate solution for people that are suffering some of such infections and of course prevent the infection of our next generations, our children. However, nothing of these will be possible without research, and of course, without funding to develop more in-depth knowledge that can be applied to find new drugs or vaccines.

Here, we selected a series of papers including reviews as well as original research on different topics of distinct important parasitic diseases affecting the world; they range since basic biology, genetics, and pathology until vaccine development as well as immune response to protozoa and helminths. Finally, it is important to say that approximately $35 \%$ of the papers received for this special issue were rejected in order to keep the quality of JBB; it is also noteworthy that more than a half of the papers published in this special issue have been already cited at least once during 2010. 


\section{Protozoan Parasites}

In this special issue, you can find studies on some of the most important protozoan infections such as malaria, amebiasis, Chagas' disease, African tripanosomiasis, toxoplasmosis, and leishmaniasis.

Amebiasis caused by Entamoeba histolytica is still a health problem in Asia and Latin America, and travelers worldwide are also affected; in their review, I. Wong-Baeza et al. "The role of lipopeptidophosphoglycan in the immune response to Entamoeba histolytica" describe the importance of lipopeptidophosphoglycan, which is recognized through TLR2 and TLR4 and leads to the release of cytokines from human monocytes, in the immune control of this infection that mainly cause diarrhea and hepatic abscess; thus lipopeptidophosphoglycan is a possible candidate molecule to develop a vaccine against amebiasis. Understanding this parasite also includes the knowledge of its genes and their function. In their original paper, I. López-Reyes et al. "Detection of the endosomal sorting complex required for transport in Entamoeba histolytica and characterization of the EhVps4 protein" found the presence and expression of endosomal sorting complexes required for intracellular transport, named EhVps4, which were related to increased phagocytosis and virulence in E. histolytica.

Another widely spread parasitic infection is toxoplasmosis, caused by the protozoa Toxoplasma gondii. Infections caused by these parasites are common and fatal in immunocompromised patients such as those infected with HIV. E. Y. Denkers "Toll-like receptor initiated host defense against Toxoplasma gondii" review recent advances in innate immunity to this parasite with special emphasis on the tolllike receptors (TLRs) which can be triggered by the parasite itself and emphasizes the complex interactions with the intestinal microbiota worsening the disease during enteric infection. Immune regulation is also a key factor during toxoplasmosis, and the research performed by E. P. Tenorio et al. "Reduction of Foxp3+ cells by depletion with the PC61 $m A b$ induces mortality in resistant $B A L B / c$ mice infected with Toxoplasma gondii" shows that ablation of $\mathrm{T}$ regs in otherwise resistant mice, induce high mortality. On the other hand, Y. Sanchez et al. "The unexpected role for the aryl hydrocarbon receptor on susceptibility to experimental toxoplasmosis" demonstrated for the first time a role for the aril hydrocarbon receptor (AHR) in a parasitic disease. They report that genetically deficient mice in AHR succumbed faster to $T$. gondii infection which was associated with an increased inflammatory cytokine response, thus revealing a role for AHR in immune regulation in toxoplasmosis.

Leishmaniasis is another wide spread parasitic infection in the world. These parasites need a vector; thus, they are transmitted by the bite of a sand fly. Several species contribute to its prevalence in all the continents. Here, we selected a series of papers that clearly show the very different sides that these infections display; the initial phase of the infection is critical where neutrophils appear to have a prominent role contributing to determine the type and magnitude of the L. major specific immune response that will develop "Charmoy et al.: The prominent role of neutrophils during the initial phase of infection by Leishmania parasites." In line with this, M. Cummings et al. "Cytokines and their STATs in cutaneous and visceral leishmaniasis" review the key importance of the signal transducer and activator of transcription (STATs) during cutaneous and visceral leishmaniasis. Interestingly, Leishmania infection is able to alter the host cell signaling in order to survive according to the original report by S. Bhardwaj et al. "Leishmania interferes with host cell signaling to devise a survival strategy." Notwithstanding, more efforts are being directed to develop new strategies to get useful vaccines "Ramírez et al.: $B A L B / c$ mice vaccinated with Leishmania major ribosomal proteins extracts combined with CPG oligodeoxynucleotides become resistant to disease caused by a secondary parasite challenge" as well as development of new drugs after detecting high resistance to the old drugs used against leishmaniasis as in "Maltezou: Drug resistance in visceral leishmaniasis."

Malaria is a tropical disease, which results in more than one million deaths annually and is caused by protozoan parasites of the genus Plasmodium and transmitted by blood-feeding Anopheline mosquitoes; effective vaccines are not available. The work by J. Schmieg et al. "A multifactorial mechanism in the superior antimalarial activity of $\alpha$-C-GalCer" demonstrates that $\alpha$-C-GalCer, that has an antimalarial activity, has a multifactorial mechanism. The in vivo administration of $\alpha$-C-GalCer induces prolonged maturation of dendritic cells (DCs), as well as an enhanced proliferative response of mouse invariant $\mathrm{V} \alpha 14$ (V $\alpha 14 \mathrm{i}) \mathrm{NKT}$ cells, both of which may also contribute to some degree to the superior activity of $\alpha$-C-GalCer in vivo. On the same line, A. Kuehn and G. Pradel "The coming-out of malaria gametocytes" reviewed the recent findings on the role of gametocytes during transmission to the mosquito and pay particular focus on the molecular mechanisms underlying gametocyte activation and emergence from the host erythrocyte during gametogenesis. Until very recently, little was known about the chromatin structure of the telomeres and subtelomeric regions in Plasmodium falciparum; that is why R. Hernandez-Rivas and collaborators "Telomeric heterochromatin in Plasmodium falciparum" discuss the telomeric heterochromatin in P. falciparum and argue that such structure may be important for telomere functions such as the silencing of the var gene family implicated in the cytoadherence and antigenic variation of these parasites. N. Basilico et al.'s "The lipid moiety of haemozoin (Malaria Pigment) and P. falciparum parasitised red blood cells bind synthetic and native endothelin-1" worked on the binding of a malaria pigment of $P$. falciparum parasitized red blood cells to a synthetic and native endothelin-1. Their findings may help understanding the consequences of parasite sequestration in severe malaria. Tomatine adjuvantation of protective immunity to a major pre-erythrocytic vaccine candidate of malaria is mediated via $\mathrm{CD} 8^{+} \mathrm{T}$-cell release of IFN- $\gamma$, according to K. G. Heal and Taylor-Robinson "Tomatine adjuvantation of protective immunity to a major pre-erythrocytic vaccine candidate of malaria is mediated via CD8+ T Cell release of IFN- $\gamma$." They conclude that further characterization of tomatine as an adjuvant in malaria vaccine development is indicated. Finally, in the same line of 
adjuvant activity, M. Legorreta-Herrera et al.s "Pretreatment with CrylAc protoxin modulates the immune response, and increases the survival of plasmodium-infected CBA/Ca mice" demonstrated that the pretreatment with CrylAc protoxin modulates the immune response of the host and increases the survival of Plasmodium-infected mice, and pointed out that by understanding how to boost innate immunity to Plasmodium infection should lead to immunological based intervention strategies.

Trypanosomiasis is caused by the protozoan Trypanosoma cruzi and T. brucei which are transmitted by insect vectors. Here, we present a series of originals works as well as reviews covering both American and African trypanosomiasis also called Chagas' disease and sleeping sickness, respectively. Many people as well as domestic and wild animals are infected or at a risk of getting these diseases by both migration and global warming. C. M. Atyame Nten et al. "Excreted/Secreted proteins from trypanosome procyclic strains" using mass spectrometry characterized almost 500 proteins secreted by T. brucei and suggested a critical role for such secretome in the virulence of this parasite. In a similar way, R. M. Corrales et al. "An experimental approach for the identification of conserved secreted proteins in Trypanosomatids" developed an experimental approach to identify conserved secreted proteins in the same parasite. In African trypanosomiasis, V. Marcoux et al. "Characterization of major surface protease homologues of Trypanosoma congolense" have characterized six major surface proteases encoded within the partially sequenced $T$. congolense genome. As the regulation and expression of such important proteins for the virulence of these parasites appears to be a critical field, we have two interesting reviews dealing with expression and regulation of genes in these protozoans: "S. MartínezCalvillo et al.: Gene expression in trypanosomatid parasites" and "C. Gomez et al.: Regulation of gene expression in protozoa parasites." In line with this, Hernández-Osorio et al.'s "Improved method for in vitro secondary amastigogenesis of Trypanosoma cruzi: morphometrical and molecular analysis of intermediate developmental forms" developed a method to analyze morphometrically and molecularly the intermediate forms of T. cruzi in vitro. Finally, regarding to immune regulation during trypanosomiasis, we have one review and one original work. T. N. Baral "Immunobiology of African trypanosomes: need of alternative interventions" wrote a very complete review regarding immunobiology of African trypanosomiasis suggesting alternate focus to improve approaches in both treatment and vaccination against trypanosomiasis. B. Espinoza et al.s "Mexican Trypanosoma cruzi I strains with different degrees of virulence induce diverse humoral and cellular immune responses in a murine experimental infection model," on the other hand, show us how different Mexican strains of T. cruzi display distinct degrees of virulence as well as induce very different cellular and humoral immune response, thus explaining part of the complexity to get successful treatment or vaccine development for this parasitic disease.

Many of the protozoan infections are transmitted by hematophagous vectors; here, these important biological agents are analyzed in three papers. The role of cysteinefree proteins in the immunobiology of arthropod-borne diseases is afforded by S. Mejia et al. "Cysteine-Free proteins in the immunobiology of arthropod-borne diseases." They discuss their findings in the context of protein structure and function, antigenicity and immunogenicity, and hostparasite relationships. In another work, R. P. Soares et al. "Differential midgut attachment of Leishmania (Viannia) braziliensis in the sand flies Lutzomyia (Nyssomyia) whitmani and Lutzomyia (Nyssomyia) intermedia" demonstrate how $L$. braziliensis display a differential attachment to the midgut of different species of its vector, the sand fly Lutzomyia. In the same context and in a letter specifically for this report, C. R. Alves et al. "The vectorial potential of lutzomyia (Nyssomyia) intermedia and lutzomyia (N.) whitmani in the transmission of leishmania (V.) braziliensis can also be related to proteins attaching" suggest that also the specific proteins involved in the attaching to the midgut of the vector may play a critical role for this infection.

\section{Helminth Infections}

Helminths are multicellular organisms which can colonize almost any tissue in their hosts. They are classified according to their adult form in nematodes (roundworms), trematodes, and cestodes (flatworms). Given the big size they can reach, the immune response against these infections is very complex. Worms infect millions of people worldwide, and they can go since inoffensive until highly dangerous and, in some cases, threatening for humans. In this special issue, we have selected research papers as well as state-of-the-art reviews covering all the three main classes of helminths.

Echinococcus multilocularis is the causative agent of alveolar echinococcosis. In their review, D. A. Vuitton and B. Gottstein "Echinococcus multilocularis and its intermediate host: a model of parasite-host interplay" discuss the immunomodulatory mechanisms involved in the pathology and protection against the larval stage (metacestodes) of this parasite and suggest the use of cytokines such as interferon$\alpha$, as well as specific antigens to treat patients in the future in order to reduce the immunopathology associated with alveolar echinococcosis and/or to prevent this very severe parasitic disease.

In the case of Taenia solium, a contribution by A. Landa et al. "Release of glycoprotein (GP1) from the tegumental surface of Taenia solium by phospholipase C from Clostridium perfringens suggests a novel protein-anchor to membranes" demonstrates that the release of glycoprotein (GP1) from the tegumental surface of its metacestode by Phospholipase C from Clostridium perfringens is a novel protein-anchor to membranes. Another aspect researched on T. solium, is the role that sex steroids plays during the development of the metacestode stage of the parasite. Particularly, progesterone in vitro treatment of the parasite induces scolex evagination and growth of the same. These effects are mediated by what looks to be a progesterone receptor of the parasite. Authors point out that this use of the hormone by the parasite 
could have strong evolutionary implications to the hostparasite relationship "Escobeda et al.: Progesterone induces scolex evagination of the human parasite Taenia solium: evolutionary implications to the host-parasite relationship." Taenia crassiceps is a parasite of rodents, and their intermediate hosts are canids. However, this parasite for a long time has been accepted to be a good alternative to develop research to give more insights of the infection produced by $T$. solium. In this issue, readers will find several papers dealing with different aspects of the infection by T. crassiceps. Firstly, P. Ostoa-Saloma et al. "Budding of Taenia crassiceps cysticerci in vitro is promoted by crowding in addition to hormonal, stress, and energy-related signals," demonstrate that budding (the asexual form of reproduction of T. crassiceps) of T. crassiceps cysticerci in vitro is promoted by crowding, in addition to hormonal, stress, and energy-related signals. On the other hand, G. Escobedo et al. "A new MAP kinase protein involved in estradiol-stimulated reproduction of the helminth parasite Taenia crassiceps" by using different approaches, including flow cytometry, confocal microscopy, and proteomics, show the process of discovery of a new protein from parasite origin, a parasite MAP kinase, that may be involved in the estradiolstimulated reproduction of this helminth parasite. Finally, A. Garza and coworkers "Substance P signaling contributes to granuloma formation in Taenia crassiceps infection, a murine model of cysticercosis" suggest that substance P signaling may contribute to granuloma formation and proinflammatory cytokine production in this infection.

Regarding Schistosoma japonicum, X. Xu et al. "Activation-induced Thelper cell death contributes to Th1/Th2 polarization following murine Schistosoma japonicum infection," demonstrate that activation-induced $\mathrm{T}$ helper cell death contributes to Th1/Th2 polarization in this infection, and suggest that S. japonicum antigen-induced Th1 and Th2 cell apoptosis involves the Th1/Th2 shift and favor both hosts and parasites. Finally, S. Hu et al. "Anti-inflammatory protein of Schistosoma japonicum directs the differentiation of the WEHI-3B JCS cells and mouse bone marrow cells to macrophages" developed a study that found an antiinflammatory protein of S. japonicum (rSj16) which directs the differentiation of the WEHI-3B JCS cells and mouse bone marrow cells to macrophages. Their results revealed that rSj16 biased the colony formation of mouse bone marrow cells towards macrophage linage.

Immunity against helminths and their interactions with the host and the intercurrent infections that they cause is presented by E. Moreau and A. Chauvin's “Immunity against helminths: interactions with the host and the intercurrent infections." They argue that studies of the immune response against helminths are of great interest in understanding interactions between the host immune system and parasites. Finally, another type of parasites and hosts that are discussed here is represented in the work by K. Rohlenová and A. Šimková "Are the immunocompetence and the presence of metazoan parasites in cyprinid fish affected by reproductive efforts of cyprinid fish?", about the immunocompetence and the presence of metazoan parasites in cyprinid fish, that could be affected by their reproductive efforts. On the same line of fish and parasites, the work by P. T. K. Woo
"Immunological and therapeutic strategies against Salmonid cryptobiosis" deals with immunological and therapeutic strategies against Salmonid cryptobiosis, caused by the haemoflagellate, Cryptobia salmositica. The disease-causing factor is a metalloprotease and a monoclonal antibody against its therapeutic. Though vaccine has been developed, only isometamidium chloride is therapeutic against the pathogen and its effectiveness is increased after conjugation to antibodies.

The early interactions between the immune system and either helminth and protozoan parasites or their derivatives have started to be a hot topic in the immunoparasitology field. In this issue, we offer four different reviews which critically show the key role played by innate cells such as dendritic cells "F. Mendlovic and A. Flisser: Dendritic cells in the gut: interaction with intestinal helminthes," "C. A. Terrazas et al.: Modulation of dendritic cell responses by parasites: a common strategy to survive," macrophages "S. J. Jenkins and J. E. Allen: Similarity and diversity in macrophage activation by nematodes, trematodes, and cestodes," and molecules such as nitric oxide against different helminth infections "A. Muro and J. L. Pérez-Arellano: Nitric oxide and respiratory helminthic diseases"; they explain how DCs did not mature after exposure to helminth antigens, as well as how macrophages are shifted towards an alternate state of activation, and pointed out how important is the induction of nitric oxide production during different helminth infections. another 2 reviews are focused on the role of these cells in both helminth and protozoan infections, for example the role of Peroxisome Proliferator-Activated Receptors (PPARs) mediating suppression of parasiticidal response is analyzed in detail by M. M. Chan et al. "Peroxisome proliferatoractivated receptor (PPAR): balance for survival in parasitic infections"; on the other hand, C. C. Stempin et al. "Arginase in parasitic infections: macrophage activation, immunosuppression, and intracellular signals," resume the role that arginase plays in promote susceptibility to protozoan and helminth infections.

\section{Using Helminths to Fight Inflammation and Autoimmunity}

The more in-depth knowledge generated in the last few years on the understanding of the immune regulation induced by helminth infections or their antigens has generated a new positive point of view regarding immune modulation by helminthes; thus, most of the helminth infections studied in more detail have showed a general mechanism that include inhibition of the immune response, low proliferative range of $\mathrm{T}$ cells and induction of regulatory cells such as Tregs, alternative activated macrophages, and "immature" DCs, which in turn may inhibited dangerous immune-reactions. Here, we offer a nice review by Y. Osada and T. Kanazawa "Parasitic helminths: new weapons against immunological disorders" suggesting that helminths can be used in a near future as weapons against autoimmune diseases. Reinforcement or support for this point of view is given by three original works using different helminth infections as regulators for 
three different pathologies, thus A. Melon's group "Infection with Hymenolepis diminuta is more effective than daily corticosteroids in blocking chemically induced colitis in mice" show how a concurrent infection with Hymenolepis diminuta is much better in reducing the pathology associated with chemically-induced colitis than classical treatment using corticosteroids. Using another cestode, Taenia crassiceps, A. Espinoza-Jiménez et al. "Taenia crassiceps infection attenuates multiple low-dose streptozotocin-induced diabetes" demonstrated for the first time how this parasite, restricted to the peritoneal cavity, was able to avoid the development of Type 1 diabetes (T1D) induced by multiple low-doses of streptozotocin. Finally, in the same order of ideas the work by P. Zaccone et al. "Immune modulation by Schistosoma mansoni antigens in NOD mice: effects on both innate and adaptive immune systems" shows how injection of soluble egg antigens from $S$. mansoni was able to induce TGF- $\beta$ from $\mathrm{T}$ cells which is important not just for Treg expansion but also for the successful Th2 response needed to reduce T1D in nonobese diabetic mice.

We hope our readers to find this first special issue of Immunobiology of Parasitic Diseases enticing and enjoy reading contributions by all authors.

Luis I. Terrazas

Abhay R. Satoskar

Jorge Morales-Montor 

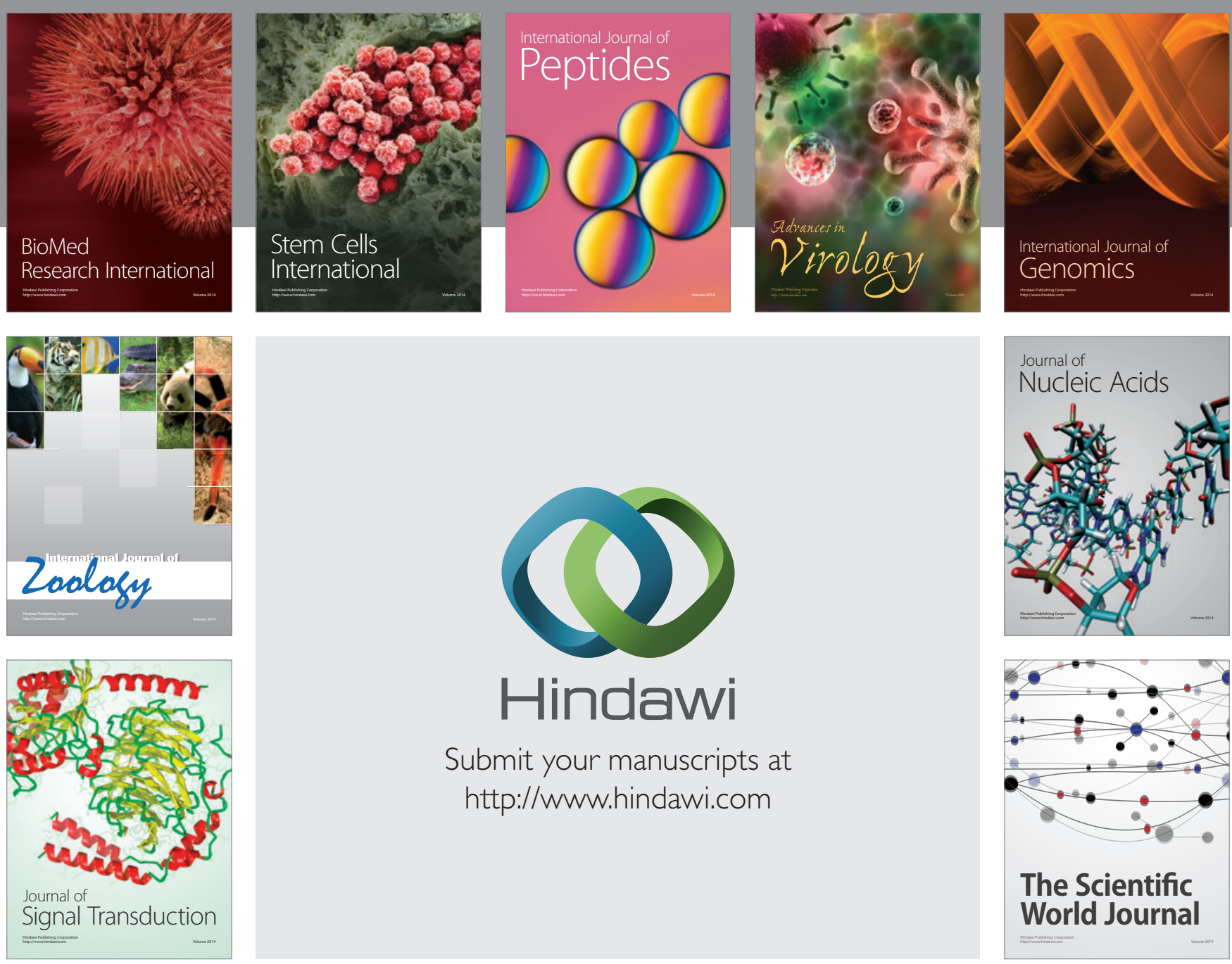

Submit your manuscripts at

http://www.hindawi.com
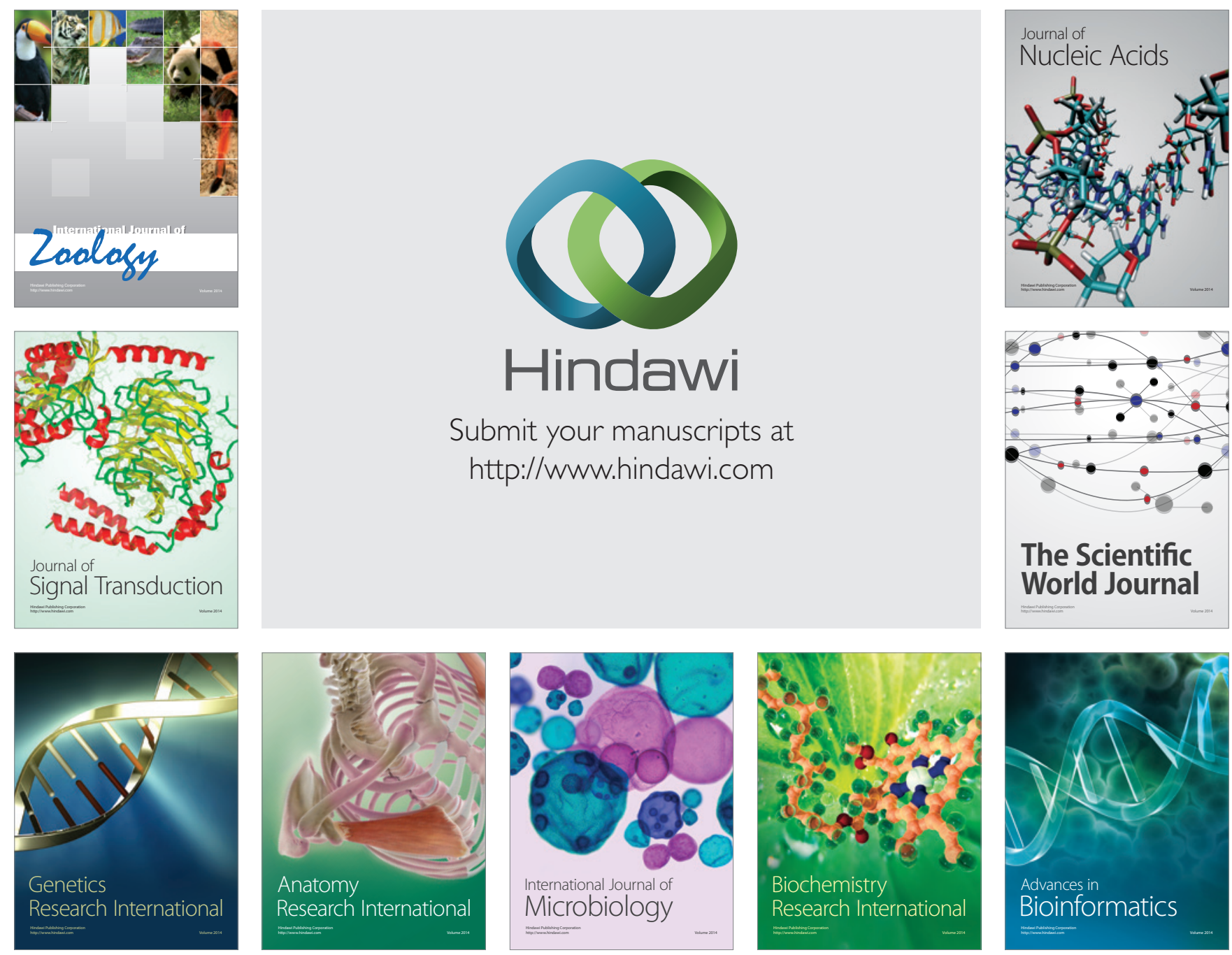

The Scientific World Journal
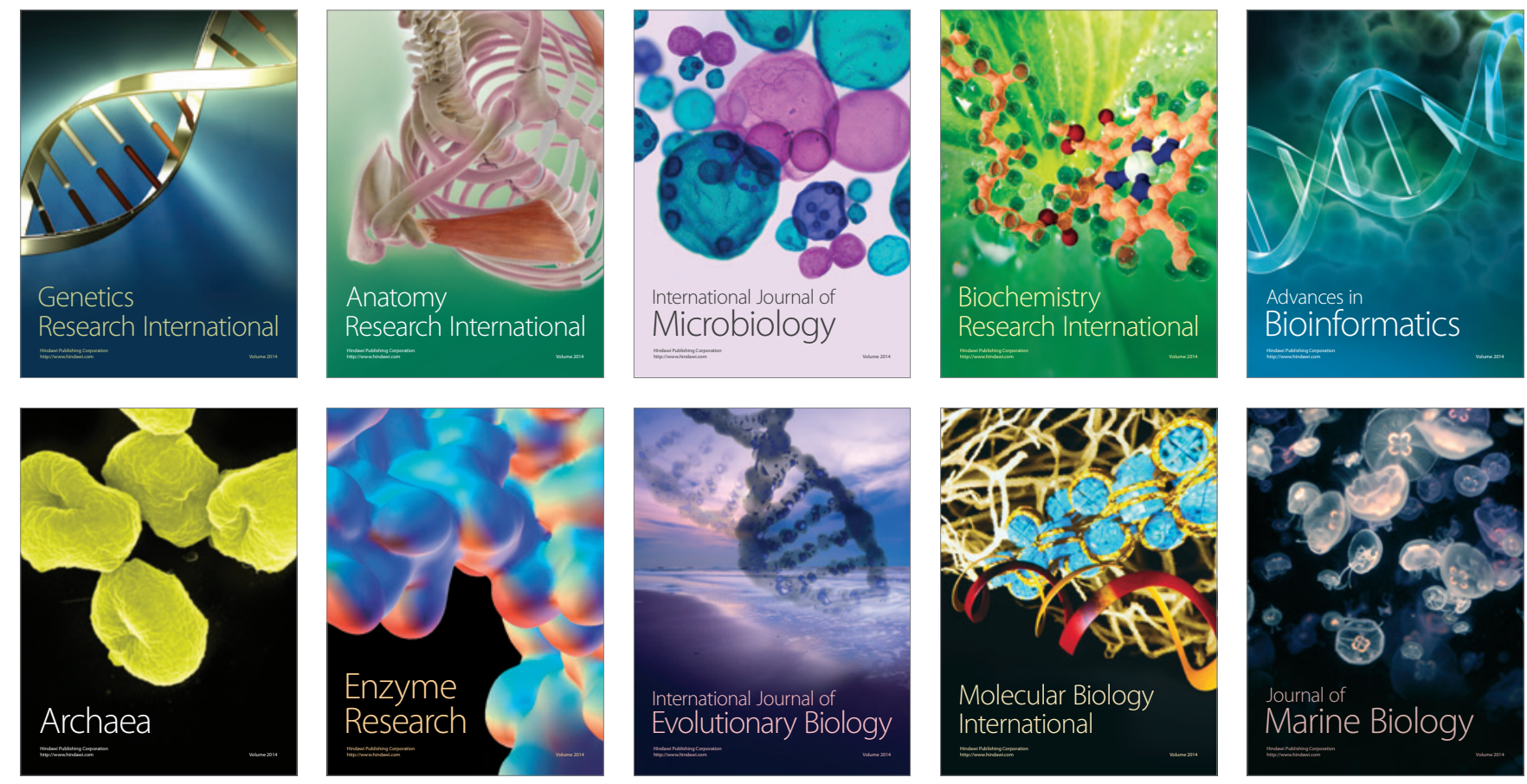